\title{
Front Matter: Volume 9343
}

, "Front Matter: Volume 9343," Proc. SPIE 9343, Laser Resonators, Microresonators, and Beam Control XVII, 934301 (16 April 2015); doi: $10.1117 / 12.2183966$

SPIE. Event: SPIE LASE, 2015, San Francisco, California, United States 


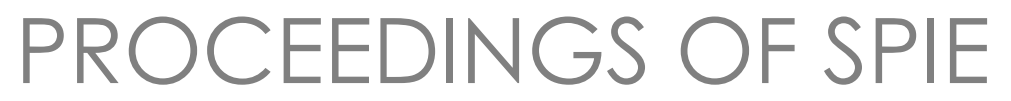

\title{
Laser Resonators, Microresonators, and Beam Control XVII
}

\author{
Alexis V. Kudryashov \\ Alan H. Paxton \\ Vladimir S. Ilchenko \\ Lutz Aschke \\ Kunihiko Washio \\ Editors
}

9-12 February 2015

San Francisco, California, United States

Sponsored and Published by

SPIE 
The papers included in this volume were part of the technical conference cited on the cover and title page. Papers were selected and subject to review by the editors and conference program committee. Some conference presentations may not be available for publication. The papers published in these proceedings reflect the work and thoughts of the authors and are published herein as submitted. The publisher is not responsible for the validity of the information or for any outcomes resulting from reliance thereon.

Please use the following format to cite material from this book:

Author(s), "Title of Paper," in Laser Resonators, Microresonators, and Beam Control XVII, edited by Alexis V. Kudryashov, Alan H. Paxton, Vladimir S. Ilchenko, Lutz Aschke, Kunihiko Washio, Proceedings of SPIE Vol. 9343 (SPIE, Bellingham, WA, 2015) Article CID Number.

ISSN: 0277-786X

ISBN: 9781628414332

Published by

SPIE

P.O. Box 10, Bellingham, Washington 98227-0010 USA

Telephone +1 3606763290 (Pacific Time) · Fax +1 3606471445

SPIE.org

Copyright (C) 2015, Society of Photo-Optical Instrumentation Engineers.

Copying of material in this book for internal or personal use, or for the internal or personal use of specific clients, beyond the fair use provisions granted by the U.S. Copyright Law is authorized by SPIE subject to payment of copying fees. The Transactional Reporting Service base fee for this volume is $\$ 18.00$ per article (or portion thereof), which should be paid directly to the Copyright Clearance Center (CCC), 222 Rosewood Drive, Danvers, MA 01923. Payment may also be made electronically through CCC Online at copyright.com. Other copying for republication, resale, advertising or promotion, or any form of systematic or multiple reproduction of any material in this book is prohibited except with permission in writing from the publisher. The CCC fee code is 0277-786X/15/\$18.00.

Printed in the United States of America.

Publication of record for individual papers is online in the SPIE Digital Library.

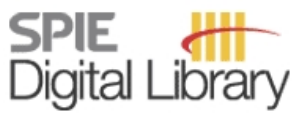

SPIEDigitalLibrary.org

Paper Numbering: Proceedings of SPIE follow an e-First publication model, with papers published first online and then in print. Papers are published as they are submitted and meet publication criteria. A unique citation identifier (CID) number is assigned to each article at the time of the first publication. Utilization of CIDs allows articles to be fully citable as soon as they are published online, and connects the same identifier to all online, print, and electronic versions of the publication. SPIE uses a six-digit CID article numbering system in which:

- The first four digits correspond to the SPIE volume number.

- The last two digits indicate publication order within the volume using a Base 36 numbering

system employing both numerals and letters. These two-number sets start with 00, 01, 02, 03, 04, $05,06,07,08,09,0 A, 0 B \ldots$. OZ, followed by 10-1Z, 20-2Z, etc.

The CID Number appears on each page of the manuscript. The complete citation is used on the first page, and an abbreviated version on subsequent pages. 


\title{
Contents
}

\author{
vii Authors \\ ix Conference Committee
}

\section{MICRORESONATOR OPTOMECHANICS I}

934302 Asymmetric response function of the transduction spectrum for a microsphere pendulum [9343-1]

MICRORESONATOR OPTOMECHANICS II

934308 Dynamical characteristics of AIGalnAs/InP microdisk lasers subject to optical injection (Invited Paper) [9343-7]

934309 Connecting microwave and optical frequencies with a vibrational degree of freedom (Invited Paper) [9343-8]

MICRORESONATOR COMBS, THZ, AND RF PHOTONICS II: JOINT SESSION WITH CONFERENCES 9343 AND 9347

9343 OE Terabit/s communications using chip-scale frequency comb sources (Invited Paper) [9343-13]

$9343 \mathrm{OF}$ Towards efficient octave-spanning comb with micro-structured crystalline resonator (Invited Paper) [9343-14]

$9343 \mathrm{OH} \quad$ Advances in Kerr optical frequency comb generation (Invited Paper) [9343-16]

\section{MICRORESONATOR SENSORS}

9343 0J Hydrogen gas sensing using palladium-coated microdisk microresonators [9343-18]

9343 OK Non-linear fluorescence excitation of Rhodamine 6G and TRITC labeled IgG in whispering gallery mode microresonators [9343-19]

$93430 \mathrm{M}$ Optically active silica and polymeric materials for microcavity lasers and sensors (Invited Paper) [9343-21]

$934300 \quad$ High frequency ultrasound detection with ultra-high-Q silica microspheres [9343-23] 
BEAM SHAPING I

9343 OP Generation of shape-invariant flat-top laser beams [9343-24]

$93430 Q \quad$ Optical trapping with superfocused high-M² laser diode beam [9343-25]

9343 OR Formation of the desired light intensity distribution on the target with bimorph deformable mirror [9343-26]

9343 OS Transverse intensity transformation by laser amplifiers [9343-27]

BEAM SHAPING II: JOINT SESSION WITH CONFERENCES 9343 AND 9356

9343 OT Optical alignment influenced aberrations in laser beam delivery systems and their correction [9343-28]

9343 OU Beam uniformity of flat top lasers [9343-29]

BEAM SHAPING III

9343 OW Minimal-effort planning of active alignment processes for beam-shaping optics [9343-31]

9343 OY FPGA-accelerated adaptive optics wavefront control part II [9343-33]

$93430 Z$ New concepts of electro-optical light deflection: EO-slab and phased EO-array [9343-34]

MICRORESONATOR FUNDAMENTALS, NOVEL TOPOLOGIES, AND DEVICES I

934310 Femtosecond laser direct writing of high-Q microresonators in glass and crystals (Invited Paper) [9343-35]

$934312 \quad$ Hybrid silicon unidirectional-emission microspiral disk lasers for optical interconnect applications (Invited Paper) [9343-37]

934314 PDMS quasi-droplet microbubble resonator [9343-39]

MICRORESONATOR FUNDAMENTALS, NOVEL TOPOLOGIES, AND DEVICES II

934315 Optimal coupling to high-Q whispering gallery modes with a sub-wavelength metallic grating coupler (Invited Paper) [9343-40]

$934316 \quad$ Strong localization of whispering gallery modes in an optical fiber via asymmetric perturbation of the translation symmetry (Invited Paper) [9343-41]

934318 Cladding modes fiber coupling to silica micro-resonators based on long period gratings [9343-43] 
$93431 \mathrm{~A} \quad$ Uncovering the physical origin of self-phasing in coupled fiber lasers (Invited Paper) [9343-45]

9343 1C Phase dynamics of high radiance fiber laser arrays with active phase control [9343-47]

9343 ID Resonator modes and mode dynamics for an external cavity-coupled laser array [9343-48]

\section{DIAGNOSTICS}

9343 IE Time-resolved deformation measurement of Yb:YAG thin disk using wavefront sensor [9343-49]

$9343 \mathrm{IF}$ Single shot $\mathbf{M}^{2}$ measurement for near infrared high energy laser pulses [9343-50]

9343 IG Characterization of vorticity in fluids by a spatially shaped laser beam [9343-51]

\section{LASER MODE CONTROL I}

934311 Analysis of thermal depolarization compensation using full vectorial beam propagation method in laser amplifiers [9343-54]

\section{LASER MODE CONTROL II}

$93431 \mathrm{M}$ Red-emitting external cavity diode laser with high slope efficiency and narrow bandwidth [9343-59]

9343 iN Wavefront control in high average-power multi-slab laser system [9343-60]

934310 Ultra stable carbon fiber high power $\mathrm{CO}_{2}$ laser with high quality laser beam and $\mathrm{AOM}$ implementation [9343-61]

\section{POSTER SESSION}

9343 IP Selective higher order fiber mode excitation using a monolithic setup of a phase plate at a fiber facet [9343-56]

$93431 Q \quad$ Investigation of the impact of fiber Bragg grating bandwidth on the efficiency of a Raman resonator [9343-62]

9343 IR Improving the intensity of a focused laser beam [9343-63]

9343 is Laser beam shape converter using spatially variable waveplate made by nanogratings inscription in fused silica [9343-64]

9343 IT Role of geometry in optothermal response of toroidal ultra-high-Q cavities [9343-65] 
$93431 \mathrm{U}$ Whispering-gallery mode lasers for biosensing: a rationale for reducing the lasing threshold [9343-66]

9343 IW High-speed transient sensing using dielectric micro-resonators [9343-69]

9343 IY Predicting the whispering gallery mode spectra of microresonators [9343-71]

934312 Manufacture of refractive and diffractive beam-shaping elements in higher quantities using glass molding technology [9343-72]

$934321 \quad$ Wavelength-tunable laser based on electro-optic effect [9343-74] 


\section{Authors}

Numbers in the index correspond to the last two digits of the six-digit citation identifier (CID) article numbering system used in Proceedings of SPIE. The first four digits reflect the volume number. Base 36 numbering is employed for the last two digits and indicates the order of articles within the volume. Numbers start with 00, 01, 02, 03, 04, 05, 06, 07, 08, 09, OA, OB...0Z, followed by 10-1Z, 20-2Z, etc.

Aceves, Alejandro B., 1D Afshar Vahid, Shahraam, 1U, 1Y

Äit-Ameur, Kamel, OP, 1R

Ali, Amir R., IW

Andrews, R. W., 09

Appelfelder, M., OY

Armani, Andrea M., 0M, 0O, $1 T$

Barry, Liam P., OE

Barth, A., OY

Beckert, E., OY

Berneschi, Simone, OK

Bischoff, Christian, $1 \mathrm{Z}$

Blöcher, Ullrich, $1 \mathrm{Z}$

Bochove, Erik J., 1C, 1D

Bohrer, Markus, 10

Bonora, Stefano, $1 \mathrm{~N}$

Braiman, Yehuda, 1C, ID

Brasch, Victor, OE

Brecher, Christian, OW

Brüning, Robert, 1P

Cagniot, Emmanuel, $1 \mathrm{R}$

Chang, Chao, OU

Chembo, Yanne K., OH

Cheng, Ya, 10

Chiang, Hung-Sheng, $1 \mathrm{~A}$

Chiavaioli, F., 18

Chistiakova, Maria V., 00

Chyla, Michal, IE

Cicak, K., 09

Collett, Oliver J. P., OS

Collier, John, IN

Cosi, F., 18

Cramer, Larry, OU

Dalton, Larry, OE

Danielson, Don, OU

Dantus, Marcos, $1 G$

Deka, N., OM

Delgado, Paul, 1C

Deryagin, A. G., OQ

Diallo, Souleymane, $\mathrm{OH}$

Diep, V., OM

Divoky, Martin, IN

Dross, Gerhard, 12

Du, Yun, 08

Dudelev, V. V., OQ

Duparré, Michael, $1 \mathrm{P}$

Elder, Delwin, OE

Endo, Akira, 1E, $1 \mathrm{~F}$

Ertel, Klaus, 1N
Eryürek, Mustafa, 0J

Fang, Wei, 10

Fang, Zhiwei, 10

Farnesi, Daniele, OK, 18

Farsari, M., OQ

Forbes, Andrew, OP

François, Alexandre, 1U, $1 Y$

Freude, Wolfgang, $\mathrm{OE}$

Fromager, Michael, OP, IR

Gertus, T., 1S

Graupeter, Thomas, 11

Grudinin, Ivan S., OF

Gu, B., 15

Gungor, E., OM

Haag, Sebastian, OW

Haas, Gil, OT

Haddadi, Sofiane, IR

Hall, Jonathan M. M., IY

Harfouche, Ali, OP, IR

Hartmann, Rainer, 11

Hasnaoui, Abdelkrim, OP, IR

Henderson, Matthew R., $1 Y$

Henry, Leanne J., 1Q

Herr, Tobias, OE

Huang, Yong-Zhen, 08

Ioppolo, Tindaro, $1 \mathrm{~W}$

Ivanenko, Mikhail, $\mathrm{OZ}$

Jain, Ravinder K., 1Q

Jelinkova, Helena, $1 \mathrm{~N}$

Ji, Hong, $1 \mathrm{U}$

Karadag, Yasin, OJ

Kemal, Juned N., OE

Kılınç, Necmettin, 0J

King, Gary, OS

Kippenberg, Tobias J., OE

Kiraz, Alper, OJ

Klopfer, Michael, 1Q

Kochkurov, L. A, 16

Koeber, Sebastian, $\mathrm{OE}$

Koochesfahani, Manoochehr, IG

Koos, Christian, $\mathrm{OE}$

Kovach, A., OM

Krasnaberski, Alexei, $\mathrm{OZ}$

Kubecek, Vaclav, IF

Kuchinskii, V. I., OQ

Kudryashov, Alexis, OR

Kuo, K., OM

Lavermann, Matthias, $\mathrm{OE}$

Lee, Jun Ho, $1 \mathrm{M}$ 
Lee, M., OM

Leger, James R., 1A

Lehnert, K. W., 09

Leuthold, Juerg, OE

Lin, Guoping, $\mathrm{OH}$

Lin, Jintian, 10

Litvin, Igor A., OP, OS

Liu, Bo-Wen, 08

Long, Heng, 08

Losch, Daniel, OW

Losev, S. N., OQ

Louhibi, Djelloul, $1 R$

Loza-Alvarez, Pablo, OK

Luan, F., 15

Lucianetti, Antonio, IN

Lylova, Ann, OR

Madugani, Ramgopal, 02

Maker, A., OM

Mann, Klaus, $1 E$

Marco, M.-Pilar, OK

Martinenghi, Romain, $\mathrm{OH}$

Mauch, S., OY

Mehrabani, S., OM

Melissinaki, $\vee ., O Q$

Merino, David, OK

Michailovas, A., is

Michailovas, K., IS

Miura, Taisuke, 1E, IF

Mocek, Tomáš, 1E, IF, IN

Monro, Tanya M., IU, IY

Müller, Tobias, OW

Na, Hong Man, IM

Nagisetty, Siva Sankar, IE, IF

Naidoo, Darryl, OP

Nair, Niketh, 1C, 1D

Neschke, Brendan, 1C

Ngcobo, Sandile, OP

Nic Chormaic, Síle, 02, 14

Norby, James, OU

Nunzi Conti, Gualtiero, 0K, 18

Ötügen, Volkan, IW

Palmer, Robert, $\mathrm{OE}$

Park, Jiyeon, $1 \mathrm{M}$

Pasquardini, Laura, OK

Pastells, Carme, OK

Pederzolli, Cecilia, OK

Peterson, R. W., 09

Petrauskienè, V., is

Pfeifle, Joerg, OE

Pflaum, Christoph, 11

Phillips, Jonathan, $1 \mathrm{~N}$

Pilar, Jan, $1 \mathrm{~N}$

Poon, Andrew W., 12

Pouya, Shahram, $1 \mathrm{G}$

Purdy, T. P., 09

Rafailov, E. U, OQ

Regal, C. A., 09

Reger, J., OY

Reinlein, C., OY

Reynolds, Tess, $1 Y$
Riesen, Nicolas, 1U, 1 Y

Righini, Giancarlo C., OK, 18

Roßmann, Jürgen, OW

Ryabtsev, Anton, $1 \mathrm{G}$

Saleh, Khaldoun, $\mathrm{OH}$

Scaggs, Michael, OT

Schindler, Philipp C., OE

Schlette, Christian, OW

Schmitt, Jana, 1 Z

Schranner, Matthias, OW

Schröter, Siegmund, IP

Schulze, Christian, IP

Severova, Patricie, $1 \mathrm{E}$

Sheldakova, Julia, OR

Shi, C., OM

Sibbett, W., OQ

Simmonds, R. W., 09

Smith, Jodie, $1 \mathrm{~N}$

Smrz, Martin, IF

Soboleva, K. K., OQ

Sokolovskii, G.S., $0 Q$

Soltani, Soheil, $1 T$

Song, Hong Joo, $1 \mathrm{M}$

Song, Jiangxin, 10

Soria Huguet, Silvia, OK, 18

Strauss, Hencharl J., OS

Sumetsky, M., 16

Tang, Jialei, 10

Tang, Suning, 21

Taşaltın, Nevin, $0 \mathrm{~J}$

Trono, C., 18

Umhofer, Udo, 1 Z

Wang, Min, 10

Ward, Jonathan M., 02, 14

Watts, Regan T., OE

Weimann, Claudius, OE

Wilde, Johannes, $1 \mathrm{P}$

Wirth, Volker, $\mathrm{OZ}$

Wolz, Michael, $1 \mathrm{Z}$

Wu, Pengfei, 21

Xiao, Jin-Long, 08

$\mathrm{Xu}$, Yingxin, 10

Yang, Yong, 02, 14

Yang, Yue-De, 08

Yu, Nan, OF

Yu, X., 15

Yun, llgu, $1 \mathrm{M}$

Zhang, Lei, 12

Zhang, Yu, 12

Zhou, Y., 15

Zontar, Daniel, OW

Zou, Ling-Xiu, 08

Zunoubi, Mohammad R., ID 


\title{
Conference Committee
}

\author{
Symposium Chairs
}

Guido Hennig, Daetwyler Graphics AG (Swtizerland)

Yongfeng Lu, University of Nebraska-Lincoln (United States)

Symposium Co-chairs

Bo Gu, Bos Photonics (United States)

Andreas Tünnermann, Fraunhofer-Institut für Angewandte Optik und Feinmechanik (Germany) and Friedrich-Schiller-Universität Jena (Germany)

Program Track Chair

Gregory J. Quarles, Optoelectronics Management Network (United States)

Conference Chairs

Alexis V. Kudryashov, Moscow State Open University (Russian Federation)

Alan H. Paxton, Air Force Research Laboratory (United States)

Vladimir S. Ilchenko, OEwaves, Inc. (United States)

Conference Co-chairs

Lutz Aschke, LIMO Lissotschenko Mikrooptik GmbH (Germany)

Kunihiko Washio, Paradigm Laser Research Ltd. (Japan)

Conference Program Committee

Andrea M. Armani, The University of Southern California (United States)

Gaurav Bahl, University of Illinois at Urbana-Champaign (United States)

Yanne K. Chembo, FEMTO-ST (France)

Jean-Claude M. Diels, The University of New Mexico (United States)

Hans Joachim Eichler, Laser- und Medizin-Technologie GmbH, Berlin (Germany)

Andrew Forbes, CSIR National Laser Center (South Africa)

Pierre Galarneau, INO (Canada)

Thomas Graf, Universität Stuttgart (Germany) 
Tobias J. Kippenberg, Ecole Polytechnique Fédérale de Lausanne (Switzerland)

James R. Leger, University of Minnesota, Twin Cities (United States)

Andrey B. Matsko, OEwaves, Inc. (United States)

Gualtiero Nunzi Conti, Istituto di Fisica Applicata Nello Carrara (Italy)

Andrew W. Poon, Hong Kong University of Science and Technology (Hong Kong, China)

Michelle L. Povinelli, The University of Southern California

(United States)

Michael J. Scaggs, Haas Laser Technologies, Inc. (United States)

Haiyin Sun, Chemlmage Corporation (United States)

Lei Xu, Fudan University (China)

Lan Yang, Washington University in St. Louis (United States)

\section{Session Chairs}

1 Microresonator Optomechanics I

Vladimir S. Ilchenko, OEwaves, Inc. (United States)

2 Microresonator Optomechanics II

Tobias J. Kippenberg, Ecole Polytechnique Fédérale de Lausanne (Switzerland)

3 Microresonator Combs, THz, and RF Photonics I: Joint Session with Conferences 9343 and 9347

Andrea M. Armani, The University of Southern California (United States)

4 Microresonator Combs, THz, and RF Photonics II: Joint Session with Conferences 9343 and 9347

Konstantin L. Vodopyanov, CREOL, The College of Optics and Photonics, University of Central Florida (United States)

Gualtiero Nunzi Conti, Istituto di Fisica Applicata Nello Carrara (Italy)

5 Microresonator Sensors

Yanne K. Chembo, FEMTO-ST (France)

6 Beam Shaping I

Michael J. Scaggs, Haas Laser Technologies, Inc. (United States)

7 Beam Delivery and Shaping: Joint Session with Conferences 9343 and 9356

Friedhelm Dorsch, TRUMPF Laser- und Systemtechnik GmbH

(Germany)

8 Beam Shaping II: Joint Session with Conferences 9343 and 9356

Kunihiko Washio, Paradigm Laser Research Ltd. (Japan) 
9 Beam Shaping III

Kunihiko Washio, Paradigm Laser Research Ltd. (Japan)

10 Microresonator Fundamentals, Novel Topologies, and Devices I Lei Xu, Fudan University (China)

11 Microresonator Fundamentals, Novel Topologies, and Devices II Andrey B. Matsko, OEwaves, Inc. (United States)

12 Coupled Resonators and Phased Arrays Alan H. Paxton, Air Force Research Laboratory (United States)

13 Diagnostics

Alexis V. Kudryashov, Moscow State Open University (Russian Federation)

14 Laser Mode Control I

James R. Leger, University of Minnesota, Twin Cities (United States)

15 Laser Mode Control II

Alan H. Paxton, Air Force Research Laboratory (United States) 
Proc. of SPIE Vol. $9343934301-12$

Downloaded From: https://www.spiedigitallibrary.org/conference-proceedings-of-spie on 26 Apr 2023 Terms of Use: https://www.spiedigitallibrary.org/terms-of-use 